\title{
Optimizing exercise interventions for men on ADT
}

D espite increasing evidence to demonstrate the benefits of physical activity for men with prostate cancer, very few of these men undertake the recommended amount of daily exercise. Two randomized controlled trials (RCTs) recently published in European Urology-representing the largest studies of their kind-have tackled the issue of how best to deliver exercise interventions to men with prostate cancer on long-term androgen deprivation therapy (ADT).

One RCT was performed by a team of investigators from across the UK, who randomized 100 sedentary men on ADT for prostate cancer to receive either a 'tapered' exercise intervention $(n=50)$ or usual care $(n=50)$. Tapering refers to the amount of supervised exercise received, whereby patients attended two supervised sessions per week for 6 weeks followed by one supervised session per week for another 6 weeks. Over the same period, self-directed exercise was increased from one session per week for the first 6 weeks, to two sessions per week for the second half of the study. Usual care involved follow-up appointments with a urologist and an oncology nurse specialist; no restrictions were placed on exercise.

Patients were assessed for healthrelated quality of life (QOL) outcomes at baseline, immediately after the exercise intervention (at 12 weeks) and at 26 weeks. Two domains of the Functional

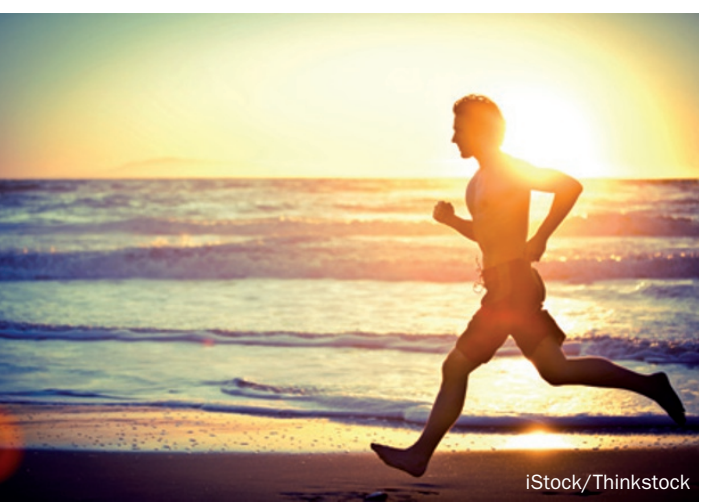

Assessment of Cancer Therapy (FACT) questionnaire were used: FACT-P to evaluate prostate-cancer-related QOL, and FACT-F to measure changes in fatigue, a major adverse effect of ADT. Both QOL outcomes were significantly better for men who received the exercise intervention compared with controls at the 12-week timepoint (a mean difference of 8.9 points for FACT-P and 5.3 points for FACT-F), and fatigue-related QOL improvement was maintained at 26 weeks (mean difference of 3.9 points). However, the benefit of exercise on disease-specific QOL was lost after supervision was withdrawn.

The investigators noted that adherence to the study also decreased after support was withdrawn and suggest that this trend might have contributed towards the loss of benefit. They suggest extension of the supervision period to improve both adherence and outcomes. Indeed, the second team of researchers had better luck with their exercise intervention, which was longer in duration. "We embarked on a multicentre and multinational exercise trial implemented over a 12 month period," explains Robert Newton, from Edith Cowan Institute, Australia. "This is the longest duration exercise intervention trial performed in this population to date."

Newton and his team evaluated an intervention model comprising 6 months of supervised exercise in the clinic followed by 6 months of home-based exercise in 50 men on ADT, compared with the provision of written guidelines and a pedometer to another 50 men. Their findings were twofold. Firstly, they demonstrated that 6 months of supervised training (two sessions of combined resistance and aerobic exercise per week) led to significant improvements over the provision of educational material for a number of outcomes. Cardiorespiratory fitness (measured as the time taken to walk 400 metres), lower body physical function (assessed with the repeated chair rise test) and muscle strength (on chest press and leg extension) were significantly improved at 6 months in men who exercised compared with controls. QOL, assessed using the Short Form-36 Health survey, was also better at 6 months for those who received structured exercise support than for those who received edcuational material (mean difference of 2.4 points).

Secondly, they found that these improvements were sustained over 6 months of self-directed exercise. "This bodes well for the long-term maintenance of health and function if participants continue the exercise programme of their own volition, even though it is outside of a formal exercise environment," Newton told Nature Reviews Urology.

The researchers would like to monitor men who follow their exercise intervention programme for even longer periods, to evaluate whether compliance extends beyond 6 months. They hope that further investigation will elucidate how exercise at home can be optimized to maximize the benefits experienced beyond the supervision period.

Taken together, the findings of these RCTs confirm that supervised exercise provides better outcomes for men with prostate cancer on ADT, and imply that longer periods of supervision might improve adherence to an exercise plan away from the clinic. Elucidating the optimal duration of supported exercise intervention for men on ADT is the logical next step. As the number of men on long-term $\mathrm{ADT}$ continues to increase, it's crucial to provide these men with the support they need to reduce their symptom burden and improve their QOL.

\section{Sarah Payton}

\footnotetext{
Original articles Bourke, L. et al. Lifestyle changes for improving disease-specific quality of life in sedentary men on long-term androgen-deprivation therapy for advanced prostate cancer: a randomised controlled trial. Eur. Urol. doi:10.1016/j.eururo.2013.09.040 | Galvão, D. A. et al. A multicentre year-long randomised controlled trial of exercise training targeting physical functioning in men with prostate cancer previously treated with androgen suppression and radiation from TROG 03.04 RADAR.
} Eur. Urol. doi:10.1016/j.eururo.2013.09.041 